\title{
Action relations, semantic relations, and familiarity of spatial position in Balint's syndrome: Crossover effects on perceptual report and on localization
}

\author{
GLYN W. HUMPHREYS, M. JANE RIDDOCH, and HELEN FORTT \\ University of Birmingham, Birmingham, England
}

\begin{abstract}
We contrasted effects of the action relationship between objects, relative to effects of semantic relation and familiarity of spatial location, in a patient with Balint's syndrome. We found enhanced perceptual report of objects placed in the correct colocations for action in comparison with when the objects (1) were placed in incorrect locations for action (Experiment 1), (2) were associatively related (Experiment 2), or (3) fell in familiar locations but were not action related (Experiment 4). In contrast, the ability to localize the objects was affected by whether the objects were in familiar as opposed to unfamiliar locations with respect to one another. Action relations between objects facilitate joint attention to both objects but do not facilitate binding to location. Familiar spatial relations facilitate spatial binding, but do not cue joint attention to the stimuli. Effects of action relation on attention and localization can be dissociated from effects of semantic relationship and familiarity of spatial location on attention and localization.
\end{abstract}

The ability to perceive, in a single instance, two objects that can be used together, may be of great benefit in a world in which our survival depends on rapidly taken actions. Recent evidence from our laboratory, based on the neuropsychological phenomenon of extinction after parietal damage, indicates that placing objects in the correct relations for action enables them to be attended together (Riddoch, Humphreys, Edwards, Baker, \& Willson, 2003). Thus, patients can show less extinction when presented with objects that are placed in the correct colocations for action than when they are given either unrelated objects or the same objects that are placed in the incorrect colocations for action. One account of this is that objects appearing in an action relation are treated as a single unit by visual attention, so that the objects no longer compete against each other, but rather cooperate in the selection process. In patients with an abnormal spatial bias in visual selection, there is typically spatial competition when two unrelated objects are presented, one on either side of fixation, with the object in the contralesional field failing to be selected (a spatial extinction effect; Karnath, 1988). However, when the objects cooperate for selection, they can then be attended together and identified. Prior studies have shown that a number of factors can lead to such

This work was supported by grants from the Medical Research Council, the Wellcome Trust, and the Stroke Association (United Kingdom). We thank G.K. for his kind participation. Correspondence concerning this article should be addressed to G. W. Humphreys, Behavioural Brain Sciences Centre, Hills Building-School of Psychology, University of Birmingham, Edgbaston, Birmingham B15 2TT, England (e-mail: g.w.humphreys@bham.ac.uk). "recovery from extinction," including low-level grouping between visual elements (Gilchrist, Humphreys, \& Riddoch, 1996; Humphreys, Romani, Olson, Riddoch, \& Duncan, 1994; Mattingley, Davis, \& Driver, 1997) and activation by both stimuli of a single stored representation (Humphreys \& Riddoch, 2003a; Kumada \& Humphreys, 2001; Ward, Goodrich, \& Driver, 1994). Objects placed in an action relation to one another typically cannot be grouped using some low-level property, so they must promote recovery from extinction through another means. This article is concerned with the factors that help to determine this effect.

There are various ways in which objects placed in an action relationship can differ from objects that do not share this relationship. One possibility is that objects in an action relation form a familiar "visual unit" because such objects commonly co-occur and/or because they commonly co-occur in this particular spatial relationship. Our visual system may come to store representations not just of single objects, but also of object pairs (or even whole scenes), in which the objects enjoy a high probability of co-occurrence in particular positions. If a pair of objects activates the same stored representation, then they can be attended as a single "perceptual unit" (Kumada \& Humphreys, 2001). A somewhat different possibility is that the representation mediating recovery from extinction is influenced not so much by the identities of the objects, but by their structural properties lending themselves to a common action. That is, the objects "afford" a common action (Gibson, 1979; Humphreys \& Riddoch, 2003a), and this affordance cues attention to both objects. According to this idea, our attentional systems are tuned not 
simply to familiar objects co-occurring, but to objects that are used together. Consider a cluttered desk. On a desk, a number of objects may co-occur (a pen, a stapler, scissors, tape, a floppy disk), only some of which may change their locations jointly, when they are used together (e.g., scissors and tape). This joint usage (in action) provides extra statistical information to help form a higher order representation, in which action-related objects are linked. This representation may also be abstracted from the particular objects involved, if the regular information that predicts the common action is not the given object ("my pair of scissors") but the consistent structural properties of objects that are used in the action (e.g., a pair of blades that can be employed in a cutting action).

These different proposals make contrasting predictions about the factors that generate recovery from extinction with object pairs. Take the idea that performance is mediated by a stored representation of particular objects. According to this view, there will be recovery from extinction for objects that are frequently found together, but not for objects that are infrequently found (and used) together. It may also be the case that what is important are not simply the object identities, but also the particular objects appearing in familiar spatial locations. These familiar colocations may have nothing to do with joint action between the objects. For example, the sun will typically be in the sky above our heads, but it will not enjoy an action relation with an object on the ground. In studies of scene perception, Biederman and colleagues (Biederman, Mezzanotte, \& Rabinowitz, 1982) have shown that objects placed in unfamiliar locations are readily noticed, suggesting that we can be sensitive to the familiar locations occupied by familiar objects in a scene. If the familiarity of the relative locations of objects is critical, then there will be recovery of extinction for objects placed in familiar relative locations, even if the objects are not action related. In contrast, if recovery from extinction is dependent on the objects affording a common action, then two objects can be jointly attended if their structural properties lend themselves to a joint action, even if the objects are not typically used together. We examined these predictions by testing for recovery from extinction with (1) familiar objects placed in familiar colocations for action (Experiment 1); (2) familiar objects that co-occur but are not necessarily found in set relative locations (Experiment 2); (3) objects that are neither frequently found nor used together but that are placed in an action relationship (Experiment 3); and (4) objects that do not enjoy an action relationship but that are typically found in common colocations (Experiment 4).

Performance was evaluated in a single case, Patient G.K. G.K. has Balint's syndrome (Cooper \& Humphreys, 2000; Hall, Humphreys, \& Cooper, 2001; Humphreys et al., 1994), a neuropsychological disorder characterized by the apparent ability to see only a single object at a time (simultanagnosia) and poor reaching to visual targets (optic ataxia). Such patients are also typically impaired at making explicit spatial judgments about objects that they can identify ("Is the cup to your left or right?"). Nevertheless, there is evidence that location information can be coded implicitly, so that the time to read spatial terms is affected by the terms' spatial congruity to the context (Cinel \& Humphreys, 2006; Robertson, Treisman, Friedman-Hill, \& Grabowecky, 1997). We have presented recent evidence that the limitation on perceiving more than one object at a time in Balint's syndrome is modulated by whether objects are placed in correct colocations for action, representing a form of recovery from extinction (Riddoch et al., 2003). The ability to identify objects presented simultaneously improved when the objects were in the correct colocations for action in comparison with when they were in incorrect spatial relations for action. Here, we attempted to untangle the factors responsible for this effect.

By testing a patient with Balint's syndrome, we were also able to address a second question, which is: What factors influence location coding in such a patient (in whom location coding is chronically impaired; see above)? Prior studies have shown that our ability to localize visual stimuli can be improved when we attend to them (Treisman \& Gelade, 1980), perhaps because one function of attention is to bind stimuli to their locations (Treisman, 1998). In this case, we might expect improved localization to go hand in hand with evidence that a patient attends to both objects present (i.e., with the factors that determine recovery from extinction). On the other hand, even if attention is cued to objects, there may still be poor explicit recovery of location information, if (for example) the spatial map that we rely on for explicit location judgments is impaired. Indeed, in an earlier study with G.K., we found exactly this. We showed that G.K. oriented spatial attention to the location of a selected object, and so was better able to detect a letter presented in the same location than a letter presented elsewhere, even though the object was explicitly mislocalized (Humphreys \& Riddoch, 2003b). Hence, being able to recover both items for report may not be sufficient for G.K. to explicitly code their locations. Nevertheless, explicit location judgments could be influenced by stored knowledge of familiar spatial relations between objects. Stored knowledge may help to bind stimuli to their locations, at least for a patient for whom this location information is abnormally noisy. If this is the case, then G.K.'s ability to localize may be improved for stimuli that occur in common relative locations, and this might hold, regardless of whether the stimuli enjoy a common action relationship. Thus, recovery from extinction might reflect a common action relationship, whereas explicit localization might reflect stored knowledge about relative object locations. This pattern of results would dissociate the factors that determine attentional selection from those that bind objects to locations.

\section{Case History}

The experiments were conducted with G.K. (born 1939), a right-handed former businessman with Balint's syndrome (Cooper \& Humphreys, 2000; Humphreys et al., 1994), who also shows a spatial preference for reporting stimuli in his right over his left visual field, when stimuli are projected bilaterally (Gilchrist et al., 1996). G.K. sustained lesions of the right medial occipital- 
parietal region, the right temporoparietal region, and the left temporoparietal region in two consecutive strokes in 1986. G.K. has simultanagnosia, optic ataxia, visual extinction, and attentional dyslexia (Hall et al., 2001). He also has some minor word-finding difficulties. However, he is well oriented in time and space and he has otherwise intact intellectual abilities. G.K. gave consent for the studies, and the experiments were given ethics approval by the School of Psychology Ethics Committee at the University of Birmingham.

\section{EXPERIMENT 1 \\ Objects With Learned Action and Position Relations}

In Experiment 1, we examined the effects on identification and localization of presenting objects that had a learned action relation and that also occupied familiar positions with respect to one another. Objects with learned action and position relations were presented either with these relations preserved (e.g., corkscrew going into a bottle) or with them violated (corkscrew going into the bottom of the bottle; see Figure 1A). When the objects were depicted in their correct spatial relationships, the objects appeared as a familiar pair falling in familiar relative locations, which afforded a common action. Each of these factors could influence performance. This provided a strong test of whether the relationship between the objects (however defined) could influence G.K.'s localization as well as his object identification. Was there not only better identification (and less extinction) of objects with preserved action/position relations (cf. Riddoch et al., 2003), but also better localization of objects even when they were identified?

\section{Method}

Procedure. G.K. was presented with pictures of single objects or pairs of objects that were positioned either in the correct colocations for action or in incorrect colocations for action (Figure 1A). On a two-object trial, one object fell in the right visual field and one in the left. On single-object trials, the stimuli fell in the same absolute positions as did the stimuli in the conditions with stimuli located either correctly or incorrectly for action. On each trial, there was a central fixation cross for $3,000 \mathrm{msec}$, viewed from $50 \mathrm{~cm}$. This was then followed by the display containing either one or two objects. G.K. was asked to identify the stimuli and then to report whether they fell in the right or left fields. There were four experimental sessions, each separated by a week. In order to generate reasonable numbers of trials in which two objects were identified, we used relatively long durations (in Sessions 1 and 2, the durations were 1,500 msec; in Sessions 3 and 4, durations of 3,000 msec were used). G.K. was also run on an additional trial block conducted 3 months after Experiments 1 and 2 (but before Experiment 3). In this session, he was presented with just the two-object stimuli from the initial sessions, and he was asked to decide whether the objects fell in positions in which they could be used together. This provided a measure of whether or not G.K. could explicitly judge the action relations between stimuli, under the current presentation conditions. In this session, the stimuli appeared for $1,500 \mathrm{msec}$. G.K. was asked to identify the stimuli on each trial, and then to state which object had fallen in his left or right visual field.
(A)
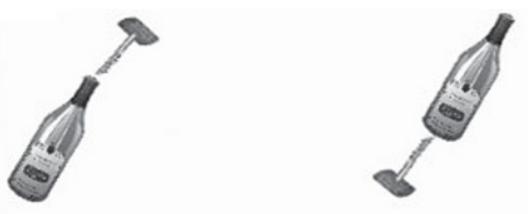

(B)
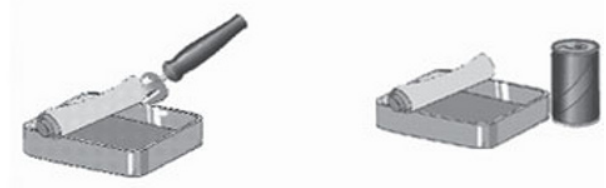

(C)
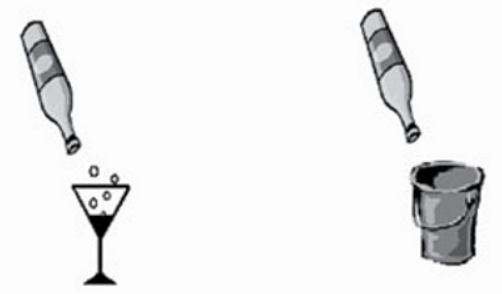

(D)
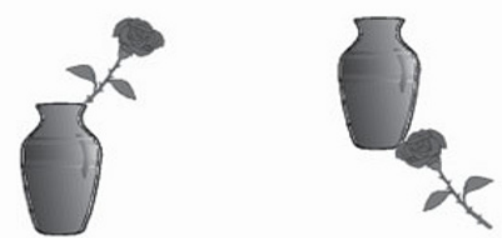

Figure 1. Example stimuli from Experiments 1 and 2. (A) Examples from Experiment 1, in which objects appeared either in correct (left) or incorrect (right) colocations for action. (B) Examples from Experiment 2. Object pairs were either action related (left) or associatively related, but not related in terms of a joint action (right). (C) Examples from Experiment 3. Objects were in either high-familiarity action pairs (left) or low-familiarity (but plausible) action pairs (right). (D) Examples of stimuli from Experiment 4 . Objects were not related by action but occupied standard locations with respect to one another. They were positioned so as to either maintain those positions or to be in unfamiliar positions.

Apparatus and Stimuli. The experiment was run on a PC, using E-Prime software (www.pstnet.com). Nine pairs of objects were typically used together, and were taken from the Corel Clipart Gallery. The stimuli were realistically colored drawings, and appeared against a white background. The items either appeared in locations appropriate for their mutual interaction or in locations inappropriate for any action. In both the correct and the incorrect location conditions, the objects appeared four times in a block, twice with Object A on the left and Object B on the right, and twice with the stimulus positions reversed by mirror reflection. This generated 72 two-object trials $(9$ pairs $\times 2$ left-right positions $\times$ correct or incorrect locations for action $\times 2$ repeats). There were also 72 one-object trials, 36 with an object on the left and 36 with an object on the right. The 144 trials in a block were presented across four test sessions conducted 
on separate weeks. The mean size of the pictures was $5.5 \mathrm{~cm}$ high $\times$ $5.9 \mathrm{~cm}$ wide, and the point of contact between pictures fell at fixation. (The same items were used in Riddoch et al., 2003.)

\section{Results and Discussion}

The results are presented in Table 1. On single-object trials, identification performance was better for right- than for left-field objects [McNemar test of change, $\chi^{2}(1)=$ $20.0, p<.01]$. On two-object trials, G.K. was more likely to identify both objects when they were in the correct spatial relations than when they were in incorrect spatial relations [McNemar test of change, $\left.\chi^{2}(1)=23.2, p<.001\right]$. These data replicate our prior results.

G.K.'s localization performance was also assessed for trials on which he identified the objects correctly. On one-object trials, his localizations were just above chance level $\left[\chi^{2}(1)=4.47, p<.05\right]$. Localization was more accurate when stimuli were in the right field $(120 / 140$ re-

Table 1

The Number of Objects Identified by G.K. in Experiments 1-3, and the Number of Correct Localization Responses Made to Objects That Were Identified Correctly

Experiment 1

Number of Objects Identified

\begin{tabular}{|c|c|c|c|}
\hline \multicolumn{2}{|c|}{ Single-Object Trials } & \multicolumn{2}{|c|}{ Two-Object Trials } \\
\hline LVF & RVF & Correct for Action & Incorrect for Action \\
\hline $\begin{array}{c}120 / 144 \\
(83 \%)\end{array}$ & $\begin{array}{c}140 / 144 \\
(97 \%)\end{array}$ & $\begin{array}{c}118 / 144 \\
(81 \%)\end{array}$ & $\begin{array}{l}80 / 144 \\
(56 \%)\end{array}$ \\
\hline \multicolumn{4}{|c|}{ Number of Identified Objects Localized } \\
\hline & & Two-C & ct Trials \\
\hline \multicolumn{2}{|c|}{ Single-Object Trials } & Correct for Action & Incorrect for Action \\
\hline \multicolumn{2}{|c|}{$\begin{array}{c}155 / 260 \\
(59 \%)\end{array}$} & $\begin{array}{c}106 / 118 \\
(90 \%)\end{array}$ & $\begin{array}{l}59 / 80 \\
(74 \%)\end{array}$ \\
\hline
\end{tabular}

Experiment 2

Number of Objects Identified

\begin{tabular}{|c|c|c|c|}
\hline \multicolumn{2}{|c|}{ Single-Object Trials } & \multicolumn{2}{|c|}{ Two-Object Trials } \\
\hline LVF & RVF & Action Related & Associatively Related \\
\hline $\begin{array}{c}138 / 150 \\
(92 \%)\end{array}$ & $\begin{array}{c}145 / 150 \\
(97 \%)\end{array}$ & $\begin{array}{l}56 / 100 \\
(56 \%)\end{array}$ & $\begin{array}{r}42 / 100 \\
(42 \%)\end{array}$ \\
\hline \multicolumn{4}{|c|}{ Number of Identified Objects Localized } \\
\hline \multirow{2}{*}{\multicolumn{2}{|c|}{ Single-Object Trials }} & \multicolumn{2}{|c|}{ Two-Object Trials } \\
\hline & & Action Related & Associatively Related \\
\hline & & $\begin{array}{l}50 / 56 \\
(89 \%)\end{array}$ & $\begin{array}{l}29 / 42 \\
(69 \%)\end{array}$ \\
\hline
\end{tabular}

Experiment 3

Number of Objects Identified

\begin{tabular}{cc}
\hline High-Familiarity Actions & Low-Familiarity Actions \\
\hline $92 / 120$ & $89 / 12$ \\
$(76 \%)$ & $(74 \%)$ \\
Number of Objects Localized \\
\hline High-Familiarity Actions & Low-Familiarity Actions \\
\hline $63 / 92$ & $42 / 89$ \\
$(68 \%)$ & $(47 \%)$ \\
& Experiment 4 \\
Number of Objects Identified \\
\hline Objects in Familiar & Objects in Unfamiliar \\
Relative Locations & Relative Locations \\
\hline $36 / 72$ & $34 / 72$ \\
$(50 \%)$ & $(47 \%)$ \\
Number of Objects Localized \\
\hline Objects in Familiar & Objects in Unfamiliar \\
Relative Locations & Relative Locations \\
\hline $31 / 36$ & $20 / 34$ \\
$(86 \%)$ & $(59 \%)$ \\
\hline
\end{tabular}


sponses, $86 \%$ correct) than when stimuli were in the left field $(35 / 120,29 \%$ correct). However, this may simply reflect a response bias to make right-side localization responses when single stimuli were reported. On two-object trials, performance was generally better, but it was reliably improved for objects in the correct spatial relations in comparison with those in incorrect relations for action $\left[\chi^{2}(1)=8.88, p<.01\right]$. However, even when objects were in the incorrect colocations for action, localization of identified objects was better than on one-object trials [summing across single objects in the left and right visual fields; $\chi^{2}(1)=5.24, p<.025$ ].

One possible reason for the localization differences, when objects were in correct and in incorrect colocations for action, is that two-object reports remained higher overall in the correct colocation condition. Even if G.K. could identify both objects when they fell in the incorrect locations for action, he may have derived a less good perceptual representation, decreasing localization accuracy. It was for this reason that we used a longer presentation duration in the later sessions ( 3 and 4), so that the identification level in the incorrect colocation condition could be matched to that in the correct colocation condition in Sessions 1 and 2. In the final block of trials, G.K.'s identification of both items in the incorrect colocation condition was 50/80 (63\%), which did not differ from his identification performance in the correct colocation condition in the first trial block $\left[51 / 80(64 \%) ; \chi^{2}<1.0\right]$. Despite this, localization remained better when objects were in the correct colocations for action compared with when they were in the incorrect colocations (51/51 vs. 37/50; Fisher exact probability, $p<.001)$. The difference in localization cannot be attributed to a difference in overall identification accuracy.

In the additional trial block in Experiment 1 (explicit judgment of correct action relations), G.K. again identified more objects when they fell in the correct locations for action [28/36 (78\%) vs. 23/36 (64\%) trials; McNemar test of change, $\left.\chi^{2}(1)=5.0, p<.05\right]$, though for objects in incorrect positions, identification performance was at least as good as in the prior trial blocks with longer durations. Nevertheless, G.K. made correct classifications of whether the objects were in the correct positions for action on only $30 / 51$ of the trials in which he identified both members of the pair. This did not differ from chance $\left(\chi^{2}<1.0\right)$.

These data confirm the results of Riddoch et al. (2003); there was better identification of objects on two-object trials when the objects fell in the correct colocations for action than when they fell in incorrect colocations. This reflected a recovery from left-side extinction. G.K. was relatively good at identifying single items in his left field under the present conditions. However, under two-object presentation conditions, he often identified only one, and this was most likely to be in his right visual field ( $70 \%$ of the errors involved report of just the right-side item). On such trials, G.K. typically failed to respond that there was anything in his left field. As we have noted, this recovery from extinction could have been due to several factors, such as the familiarity of the objects as a pair (occurring in familiar locations with respect to one another), the fact that the objects were commonly used together and were in the correct colocations for action, or the fact that they could be used together and were in the appropriate locations for a common action.

The data also show that, whichever factor was important, G.K. was also better able to localize objects when they appeared in correct colocations than when they appeared in incorrect (novel) colocations. Note that, in both the correct and the incorrect colocation conditions, each object was equally likely to fall in the left and right visual field, so the fact that they appeared in familiar relative spatial locations was not sufficient for him to be able to report which object fell in which field. Localizations were also more accurate on two-object trials than on one-object trials, even when G.K. could identify the stimuli. One possible account of this is that a form of relative-position coding took place on two-object trials that supported localization even if the objects were not tightly bound to their absolute spatial positions. In contrast, localization on one-object trials may have depended on coding absolute position with respect to fixation, a task that is very difficult for G.K. (Humphreys \& Riddoch, 2003b; Humphreys et al., 1994). Note, though, that relative-position coding should have been equivalent in the two-object conditions, so the benefit for action-related objects cannot be attributed to this.

\section{EXPERIMENT 2 Action-Related Versus Associatively Related Objects}

Riddoch et al. (2003) demonstrated that patients showing extinction were better at identifying two objects placed in a familiar action relationship with respect to one another than two objects that were associatively but not action related (can and tin, vs. tin-opener and tin). Here, we attempted to replicate this result with G.K., but also assessed whether any effects occurred not only for object identification but also for localization.

\section{Method}

In Experiment 2, we compared G.K.'s ability to identify and localize stimuli that were either objects that would normally be used together (placed in the correct colocations for action) or objects that were associatively related but not typically used together. The associatively related objects were chosen to have higher levels of verbal association than the action-related objects, and the action-related and associatively related objects were rated as equally visually familiar as pairs (see below). The individual stimuli used on twoobject trials were also presented on single-object trials. Examples of the stimuli are presented in Figure 1B. The stimuli were presented in five sessions; durations of 1,500 msec were used in Sessions 1-3 and durations of 3,000 msec in Sessions 4 and 5.

Two-object trials contained objects that were either used together in action or were verbally associated. There were 10 pairs of action-related objects and 10 pairs of associatively related objects, each based around a common set of 10 target objects. (These 10 objects were paired with 10 other action-related and 10 other associatively related stimuli to create the two conditions.) In one session, 
each object pair appeared twice, once with Object A on the left and Object B on the right, and once with the stimuli mirror-reflected (20 trials for both the action- and the associatively related conditions). There were also 60 single-item trials, 30 left and 30 right (for each object in the related pairs) per session. Nine independent participants rated the visual familiarity of each object pairing ("How visually familiar do you judge these objects as a pair? A visually familiar pair is a pair you feel you have encountered frequently; a visually unfamiliar pair is a pair you feel you have not encountered together very frequently"). The mean familiarity rating for the action-related pairs was $4.2(1=$ unfamiliar, $5=$ very familiar $)$ and it was 4.0 for the associatively related pairs $(F<1.0)$. The stimuli were derived from the Birkbeck Word Association norms. Association ratings were 32.2\% for the associated items and $1.9 \%$ for $6 / 10$ action-related pairs (only 6 action-related pairs were listed, itself an indication that these objects were not judged as close verbal associates); the associatively related objects were rated as being more associatively related than the action-related pairs $[F(1,14)=26.3, p<.0001]$.

We also had a group of 10 independent participants rate the degree of action relation between the stimuli. For this, we asked them to give a high rating to "two stimuli that were depicted as being used directly together, with one in motion, changing the state in the other." To emphasize that participants should rate the "affordance" and not simply the familiarity of objects being used together, we gave, as an example of a strong action pairing, stirring a glass of water with a stick; these are two objects that could be used directly to change the state of one of them, but they are not a very familiar action pairing. The action-related pairs were given a mean action rating of 6.5 $(S D=0.7$, scale of 1 to 7 , where 7 is high), whereas the associatively related stimuli had a mean action rating of $2.0(S D=1.1)$. The action-related stimuli were indeed given higher action ratings than were the associatively related items $[t(18)=11.21, p<.001]$.

The mean size of the pictures was $5.4 \mathrm{~cm}$ wide $\times 5.8 \mathrm{~cm}$ high. Each object pair was presented twice in a session, once with Object $A$ on the left and Object B on the right, and the other time with the object locations reversed (20 trials in each condition in a session). There were also one-object trials in which either each target object or its action- or associatively related partner appeared, once in the left visual field and once in the right (30 trials with one object in the left field and 30 with one in the right field, in one session).

\section{Results and Discussion}

On single-object trials, there was a nonsignificant trend for identification performance to be better for objects in G.K.'s right field, relative to his left visual field [ $\mathrm{McNe}-$ mar test, $\left.\chi^{2}(1)=2.24, p>.05\right]$. On two-object trials, there was better identification for the action-related objects than for the associatively related objects $\left[\chi^{2}(1)=\right.$ $3.92, p<.05]$, matching the pattern found in prior data (Riddoch et al., 2003).

Localization responses were scored for trials in which G.K. correctly identified the stimuli. On one-object trials, G.K.'s localizations were borderline different from chance $\left[\chi^{2}(1)=3.76, p=.052\right]$. As in Experiment 1, he localized right-side objects better than left-side objects (114/145 [79\%] vs. 50/138 [36\% correct]), which may reflect better right-side localization or a response bias. On two-object trials, there was better localization of identified objects in action-related than in associatively related pairs $\left[\chi^{2}(1)=6.29, p<.025\right]$. The trend for better localization with associatively related pairs than with single objects was not reliable $\left[\chi^{2}(1)=1.87, p>.05\right]$. To guard against localization's being better for action-related pairs than for associatively related pairs because identification tended to be better, we also compared localization for actionrelated pairs in Sessions 1-3 (duration: 1,500 $\mathrm{msec}$ ) with localization for associatively related pairs in Sessions 4 and 5 (duration: 3,000 msec). Identification accuracy was then matched in the two conditions (action related 27/60 [45\%], associatively related 19/40 [48\%]); nevertheless, there remained an advantage for localization accuracy for action-related pairs (25/27 [93\%]) over associatively related pairs $\left[13 / 19(68 \%) ; \chi^{2}(1)=4.54, p<.05\right]$.

There was an advantage for both identification and localization for pairs of objects that were action related in comparison with objects that were rated as being associatively (but not action) related. The localization advantage was not simply due to the overall better level of perceptual report for action-related stimuli, because it occurred across blocks in which identification performance was equated in the two critical conditions. The advantages in identification and localization could be because of the action relations between the stimuli, or they could be because, in the action- but not the associatively related pairs, the objects also occupied familiar spatial positions with respect to one another. (Or possibly both factors are important; perhaps one factor influences identification, and the other localization.) In the associatively related condition, though the objects were strongly associated with one another, they did not have set spatial locations with respect to one another. These different factors are teased apart in Experiments 3 and 4.

\section{EXPERIMENT 3 Objects That Are Frequently or Infrequently Used Together}

In Experiment 3, we contrasted identification and localization for pairs of objects that were either frequently used together (wine bottle and glass) or infrequently used together (wine bottle and bucket) but could be used together (i.e., pairs of objects whose structural properties afforded a common action). If knowledge about particular objects, or about the relative locations these objects typically occupy with respect to one another is stored, then performance should be better with the objects that are frequently used together than with those that are not.

\section{Method}

In Experiment 3, G.K. was presented with only two-object trials always containing pictures of objects that were correctly positioned and could be used for action. However, the objects were either frequently used together (wine bottle pouring into a wine glass), or infrequently used together (wine bottle pouring into a bucket; Figure 1C). The stimuli were displayed for $3,000 \mathrm{msec}$.

With the stimuli used in Experiment 3, 10 independent observers were asked to rate how frequently the objects in each pair were used together $(10=$ highly likely to be used together, $1=$ highly unlikely to be used together). The high-frequency action pairs were given a mean rating of 9.7 , and the low-frequency action pairs were given a rating of 3.8. The objects in the high-frequency action pairs were rated as being used together more often than the low-frequency action pairs $[F(1,9)=150.6, p<.0001]$. We also asked the same 10 independent participants who took part in Experiment 2 to rate whether the objects were action related. The mean ratings for the 
high- and low-familiarity action pairs were $6.5(S D=0.8)$ and 6.1 $(S D=0.7)$ respectively, and the ratings for the two categories did not differ reliably $[t(18)=1.24, p>.05]$.

The mean size of the items was $2.7 \times 0.9 \mathrm{~cm}$ for targets; the mean size partner in the high-familiarity action pairs was $1.9 \times 1.1 \mathrm{~cm}$, and for the low-familiarity action pairs it was $1.8 \times 1.4 \mathrm{~cm}$. Only two-item trials were presented. There were 10 pairs of objects that were frequently used together and 10 pairs that could be used together, though this typically would not be the case. One target object was common to each pair (e.g., the wine bottle, in the example above), and the other was chosen according to the action condition being manipulated. Each pair of objects was shown six times with the common object on the right and its partner on the left, and six times with the common object on the left and its partner on the right (120 trials per condition).

\section{Results and Discussion}

G.K. identified 92/120 (76\%) of the objects in the highfamiliarity action condition and 89/120 (74\%) in the lowfamiliarity action condition. There was no advantage in terms of identification accuracy for the high-familiarity actions $\left(\chi^{2}<1.0\right)$. Of the objects identified, G.K. localized $63 / 92(68 \%)$ of the objects in the high-familiarity action pairs but only $42 / 89$ (47\%) of the low-familiarity action objects. Localization of high-familiarity pairs was better than localization of low-familiarity pairs $\left[\chi^{2}(1)=\right.$ $8.42, p<.01]$.

There was no benefit in identification for objects that were frequently used together and placed in the correct colocations for action, in comparison with objects that were infrequently used together but that were nevertheless positioned for a common action. This was not because our manipulation of the frequency of the action was not sufficiently strong. When localization of correctly identified objects was examined, there was an advantage for objects that were frequently used together. These data suggest a contrasting pattern of data for the identification and localization measures. Identification was unaffected by whether the objects were frequently used together or not, though both our prior work and Experiments 1 and 2 here indicate that identification is sensitive to the objects' being placed in appropriate colocations for action. In contrast, localization does appear to have been sensitive either to the frequency of the action with the particular objects, or to whether the particular objects fell in standard locations with respect to one another (since the high- and low-frequency action pairs here differed on both counts). Which of these factors was crucial was evaluated in Experiment 4 , in which we presented G.K. with objects that were not action related, but that did occupy standard, realworld locations with respect to one another.

\section{EXPERIMENT 4 Objects That Occupy Standard Spatial Locations With Respect to One Another}

In Experiment 4, G.K. was presented with objects that typically occupy consistent spatial relationships when they appear in the real world, but, critically, the objects were not depicted so that one object appeared to be in an unstable state and to be acting on the other (in Figure 1D, the flower is in a stable location with regard to the vase; in contrast, in Figures 1A through 1C, the acting stimulus is in an unstable pose, in the state of acting on the other object). In one condition, the objects fell in the correct spatial positions with respect to one another; in a second condition, the objects were shifted so that they no longer occupied these standard locations.

\section{Method}

In Experiment 4, we again used only two-object trials, but this time we presented stimuli that could occupy familiar positions in relation to one another (e.g., a rose and a vase). The objects were displayed either in these familiar colocations (the rose above, emerging either to the left or right of the vase) or in unfamiliar colocations (the rose below, either to the left or right of the vase; Figure 1D). We again used a duration of $3,000 \mathrm{msec}$.

The objects were chosen solely on the basis of whether they typically occupied set positions with respect to one another when they occurred in the real world. A group of 10 independent participants rated the degree of action relation between the objects (see Experiment 2). The average action rating for objects in familiar colocations was $2.0(S D=1.1)$, whereas that for objects in unfamiliar colocations was $1.8(S D=0.9)$. These two ratings did not differ $(t<1.0)$. The rated action relations between the stimuli in familiar colocations here did not differ from those given to associatively related objects in Experiment $2(t<1.0)$, and they were lower than the ratings given to the action-related objects in Experiments $2[t(17)=10.61, p<$ .0001 ] and 3 [for the comparison with high-familiarity action pairs, $t(17)=10.35$; for the comparison with low-familiarity action pairs, $t(17)=9.53$, both $p \mathrm{~s}<.001]$.

There were nine pairs of objects, which were each shown four times in one block. Objects were displayed either in locations that were consistent with the colocations of the stimuli in real life (the rose above the vase) or in locations that violated this real-life relationship (the rose below the vase). In both the consistent and inconsistent conditions, a stimulus pair was shown once with Stimulus A on the left and Stimulus B on the right, and once with A on the right and $\mathrm{B}$ on the left. There were four blocks of trials. The items were the same size as in Experiment 3. Only two-object trials were presented.

\section{Results and Discussion}

G.K. identified $36 / 72(50 \%)$ of the paired objects in the correct location condition, and 34/72 (47\%) of the objects in the incorrect location condition. Performance did not differ in the two conditions $\left(\chi^{2}<1.0\right)$. When the objects fell in the correct relative locations, 31 of the 36 identified pairs were localized (86\%), whereas only $20 / 34$ $(59 \%)$ of the pairs were localized when the objects fell in nonstandard relative locations. There was an advantage in localization for objects placed in their standard relative locations, relative to objects placed in novel relative locations $\left[\chi^{2}(1)=6.58, p<.01\right]$. Performance with objects placed in novel locations did not differ from chance $\left(\chi^{2}<\right.$ $1.0)$.

We also compared G.K.'s identification of objects in the correct location condition here with his identification of action-related objects in Experiments 2 and 3, for trials in which equivalent exposure durations were used. Identification accuracy was higher for action-related objects in Experiment 2 (29/40 correct, with a 3,000 msec exposure) 
than for objects in the correct locations in Experiment 3 $\left[\chi^{2}(1)=5.35, p<.025\right]$, and accuracy was also higher than in the latter condition in the high-familiarity and low-familiarity action-related conditions of Experiment 3 $\left[\chi^{2}(1)=14.4\right.$ and 11.56 , both $\left.p s<.001\right]$. In contrast, relative to the action-related conditions in Experiment 3, objects that were identified were more likely to be localized correctly in the correct location condition of Experiment 4 $\left[\chi^{2}(1)=4.14\right.$ and 15.98 , vs. the familiar and unfamiliar action-related pairs].

The data from Experiment 4 converge with those from Experiment 3 in suggesting that G.K.'s ability to localize objects is affected by stored knowledge that the objects typically occupy set spatial positions with respect to one another. This ability seems separate from any effect of action relations on performance, since the stimuli used here did not share an action relationship and were rated as having a weaker action relationship than did the action-related objects used in Experiments 2 and 3. Indeed, localization for objects in correct colocations was more accurate than localization for objects in the action-related conditions of Experiment 3. In contrast, identification performance was worse for objects here than in the action-related conditions of Experiment 3, and identification was unaffected by whether the objects enjoyed a standard spatial relationship in the world. Thus, identification appeared to be unaffected by whether objects occupied familiar locations with respect to one another. A similar conclusion can be drawn from Experiment 3, in which identification was not better for objects used frequently together (and so occupying consistent relative positions) than for objects that are used infrequently and do not occupy consistent positions with respect to one another.

\section{GENERAL DISCUSSION}

\section{Recovery From Extinction}

As in our prior research with patients showing extinction, there was better identification of two objects when they could be used together and fell in the correct colocations for action, relative to when they fell in the incorrect colocations for action (Experiment 1) or when they were associatively but not action related (Experiment 2). The data presented here advance these results, since they show no difference between the identification of objects that were in a high-frequency action pair and those that were in a low-frequency pair that nevertheless could be used together (Experiment 3). It seems that presenting objects in the correct colocations for action, even if the objects are used together infrequently, is sufficient to facilitate recovery from extinction. In Experiment 4, we also found no advantage for identifying objects that standardly appear in set positions relative to one another, in comparison with objects presented in novel positions, and identification performance here was worse than for action-related objects in Experiment 3. We conclude that the effects of action relations on identification are not due to the familiarity of object positions per se, but rather due to the affordance offered by objects having structural properties that are appropriate for a common action. When G.K. was unable to identify both of the objects present on a trial, he typically reported the presence of just one item. The effect of an action relation between the objects, then, seems to be to cue attention to both of the stimuli, reducing the extinction effect that is otherwise apparent. A more precise analysis of the critical factors, though, requires further work. For example, from the present results, we do not know whether implied motion is necessary to produce the effect, or whether it is sufficient that one object can be used to change the state of the other. This can be assessed by varying the presence of implied motion between the objects.

The results are consistent with other findings demonstrating that stimuli subject to extinction nevertheless undergo some degree of information processing, so that (for example) they can enter into grouping relations with items in the ipsilesional field (Gilchrist et al., 1996). Here, extinguished items are processed sufficiently to enable their structural properties to be coded as part of an action event with the ipsilesional stimulus. We suggest that visual attention is sensitive to such action events, in such a way that attention spreads across objects participating in the event. In patients with parietal lesions and a spatial bias in selection, the spread of attention to both members of an action event counteracts the spatial bias, facilitating their ability to identify both objects on a trial.

\section{The Neural Basis of Action Effects of Attention}

We have found that the action relations between objects reduce extinction in patients with both unilateral (Riddoch et al., 2003) and bilateral parietal damage (the present work). The neural basis of this effect remains unclear at present. For example, there is considerable evidence from functional imaging studies that action-related properties of individual objects activate regions of inferior parietal and frontal cortices, particularly in the left hemisphere (Grèzes \& Decety, 2002; Grèzes, Tucker, Armony, Ellis, \& Passingham, 2003; Rumiati et al., 2004). Accordingly, one might speculate that action relations cue attention through activation of neurons in these regions that respond to actions created by pairs rather than single objects. In cases in which the lesions overlap the regions identified in imaging studies (see Riddoch et al., 2003), there may still be some increased activation to action-related pairs, from residual neurons preserved in the area. In the patients, this extra activation may be sufficient to cue attention to both objects present, ameliorating the spatial bias in attention induced by the lesion. However, there is also evidence for relatively ventral activation, in the inferior occipitotemporal cortex, when participants make decisions about the kinds of actions they would perform with objects (Phillips, Humphreys, Noppeney, \& Price, 2002). An alternative account of the neuropsychological data, then, is that recovery from extinction is mediated by activation of these ventral areas responding to visual properties of objects linked to action. More definitive conclusions on 
these issues will require functional imaging studies with the patients.

\section{Object Localization}

In addition to effects of an action relation on performance, we have also shown that G.K.'s ability to localize stimuli is influenced by whether the stimuli typically occupy set spatial positions with respect to one another, in the real world. Although his localization ability was highly impaired, he performed better when objects typically had set spatial locations. We can rule out various accounts of this result. For example, the effect was not a reflection of the better identification of objects when they fell in learned spatial locations with respect to one another, since it occurred even when identification levels were matched in conditions in which objects were and were not located in these positions (Experiments 1 and 2). It was also not produced by G.K.'s identifying the objects and then guessing which one fell on the left and which on the right side of fixation, since the objects were equally likely in either field, both when they occupied standard positions and when they fell in novel locations. Another alternative account is that, in Experiment 1, the better localization of objects in the correct relative to the incorrect locations may have been because G.K. was able to use information from individual objects in each pair to guess the location of its partner. For example, after perceiving the wine bottle in Figure 1A as being upright and turned to the right, G.K. may have guessed that the corkscrew was on the right rather than the left side. However, this explanation fails to account for the results from Experiments 2 and 3, in which objects were always shown in the same locations in the critical conditions (e.g., for action-related vs. associatively related pairs, or for highor low-familiarity pairs). If the orientation of the bottle, in Figure 1C, was used to infer the location of its partner, then this should have occurred with low- as well as highfamiliarity actions. However, we found that localization was better for high-familiarity action pairs. This last result suggests that, rather than guessing the relative locations from individual objects, G.K. uses learned action relations between two objects to help him "bind" objects to an internal representation of space that was damaged following his parietal cortex lesions, perhaps generating unstable representations (Cinel \& Humphreys, 2006). In general, the use of stored knowledge to help bind objects to locations may be used to guide processes such as scanning, in the real world, when we encounter familiar environments. Remarkably, the advantage for objects placed in the correct colocations for action arose even though G.K. performed at no better than chance when asked to discriminate explicitly whether objects were correctly positioned for action, in Experiment 1.

We also noted that, in addition to the differences we observed in the two-object conditions, accuracy tended to be better when two objects were presented than it was on one-object trials. One possibility here is that a form of relative-position coding took place on two-object trials that supported localization even if the objects were not tightly bound to their absolute spatial positions. However, localization on one-object trials may have depended on coding absolute position with respect to fixation, a task that is very difficult for G.K. (Humphreys \& Riddoch, 2003b; Humphreys et al., 1994). Note, however, that relative-position coding should have been equivalent in all of the two-object conditions, so the benefit for actionrelated objects cannot be attributed to this.

At least two main accounts have been offered for how visual representations might be bound to their spatial positions. According to feature integration theory (Treisman, 1988), object and spatial representations are bound by focusing spatial attention on a location map for the stimuli, filtering out other items so that only attended features are bound together. According to a temporal binding account (e.g., Singer \& Gray, 1995), objects may be bound to their spatial locations by temporal synchrony in the firing of object- and space-coding neurons. For feature integration theory, bilateral parietal damage disrupts performance by damaging the map needed for spatial filtering; for the temporal binding account, bilateral parietal damage may be disruptive because it prevents feedback signals that help to synchronize neural firing. Under either of these accounts, learned position relations could facilitate binding if stored knowledge about object position stabilizes parietal activity supporting either spatial coding or temporal synchrony in neural firing.

\section{REFERENC ES}

Biederman, I., Mezzanotte, R. J., \& Rabinowitz, J. C. (1982). Scene perception: Detecting and judging objects undergoing relational violations. Cognitive Psychology, 14, 143-177.

Cinel, C., \& Humphreys, G. W. (2006). On the relations between implicit and explicit spatial binding: Evidence from Balint's syndrome. Cognitive, Affective, \& Behavioral Neuroscience, 6, 127-140.

Cooper, A. C. G., \& Humphreys, G. W. (2000). Coding space within but not between objects: Evidence from Balint's syndrome. Neuropsychologia, 38, 723-733.

GiBson, J. J. (1979). The ecological approach to visual perception. Boston: Houghton Mifflin.

Gilchrist, I. D., Humphreys, G. W., \& Riddoch, M. J. (1996). Grouping and extinction: Evidence for low-level modulation of visual selection. Cognitive Neuropsychology, 13, 1223-1249.

Grèzes, J., \& Decety, J. (2002). Does visual perception of object afford action? Evidence from a neuroimaging study. Neuropsychologia, 40, 212-222.

Grèzes, J., Tucker, M., Armony, J., Ellis, R., \& Passingham, R. E. (2003). Objects automatically potentiate action: An fMRI study of implicit processing. European Journal of Neuroscience, 17, 2735-2740.

Hall, D. A., Humphreys, G. W., \& Cooper, A. C. G. (2001). Neuropsychological evidence for case-specific reading: Multi-letter units in visual word recognition. Quarterly Journal of Experimental Psychology, 54A, 439-467.

Humphreys, G. W., \& RidDoch, M. J. (2003a). From vision to action and action to vision: A convergent route approach to vision, action, and attention. In D. E. Irwin \& B. H. Ross (Eds.), Cognitive vision: The psychology of learning and motivation. (Vol. 42, pp. 226-264). New York: Academic Press.

HumphreYs, G. W., \& RidDOCH, M. J. (2003b). From what to where: Neuropsychological evidence for implicit interactions between objectand space-based attention. Psychological Science, 14, 487-492.

Humphreys, G. W., Romani, C., Olson, A., Riddoch, M. J., \& Dun- 
CAN, J. (1994). Non-spatial extinction following lesions of the parietal lobe in humans. Nature, 372, 357-359.

KarNath, H.-O. (1988). Deficits of attention in acute and recovered visual hemi-neglect. Neuropsychologia, 26, 27-43.

Kumada, T., \& Humphreys, G. W. (2001). Lexical recovery from extinction: Interactions between visual form and stored knowledge modulate visual selection. Cognitive Neuropsychology, 18, 465-478.

Mattingley, J. B., Davis, G., \& Driver, J. (1997). Preattentive fillingin of visual surfaces in parietal extinction. Science, 275, 671-674.

Phillips, J., Humphreys, G. W., Noppeney, U., \& Price, C. J. (2002). The neural substrates of action retrieval: An examination of semantic and visual routes to action. Visual Cognition, 9, 662-684.

Riddoch, M. J., Humphreys, G. W., Edwards, S., BaKer, T., \& Willson, K. (2003). Actions glue objects but associations glue words: Neuropsychological evidence for multiple object selection. Nature Neuroscience, 6, 82-89.

Robertson, L., Treisman, A., Friedman-Hill, S., \& Grabowecky, M. (1997). The interaction of spatial and object pathways: Evidence from Balint's syndrome. Journal of Cognitive Neuroscience, 9, 295-317.

Rumiati, R. I., Weiss, P. H., Shallice, T., Оtтoboni, G., Noth, J.,
ZILLES, K., \& FINK, G. R. (2004). Neural basis of pantomiming the use of visually presented objects. NeuroImage, 21, 1224-1231.

Singer, W., \& GRAY, C. M. (1995). Visual feature integration and the temporal correlation hypothesis. Annual Review of Neuroscience, 18, 555-586.

Treisman, A. (1988). Features and objects: The Fourteenth Bartlett Memorial Lecture. Quarterly Journal of Experimental Psychology, 40A, 201-237.

Treisman, A. (1998). Feature binding, attention and object perception. Philosophical Transactions of the Royal Society of London: Series B, 353, 1295-1306.

Treisman, A., \& Gelade, G. (1980). A feature-integration theory of attention. Cognitive Psychology, 12, 97-136.

WARD, R., GoODRICH, S., \& DRIVER, J. (1994). Grouping reduces visual extinction: Neuropsychological evidence for weight-linkage in visual selection. Visual Cognition, 1, 101-129.

(Manuscript submitted April 27, 2005; revision accepted for publication March 13, 2006.) 